\title{
Fear of eyes: Triadic relation among social anxiety, trypophobia, and discomfort for eye cluster
}

Kengo Chaya, Yuting Xue, Yusuke Uto, Qirui Yao, Yuki Yamada

Imagine you are being gazed at by multiple individuals simultaneously. Is the provoked anxiety a learned social-specific response or related to a pathological disorder known as trypophobia? A previous study revealed that spectral properties of images induced aversive reactions in observers with trypophobia. However, it is not clear whether individual differences such as social anxiety traits are related to the discomfort associated with trypophobic images. To investigate this issue, we conducted two experiments with social anxiety and trypophobia and images of eyes and faces. In Experiment 1, participants completed a social anxiety scale and trypophobia questionnaire before evaluation of the discomfort experienced upon exposure to pictures of eye. The results showed that social anxiety had a significant indirect effect on the discomfort associated with the eye clusters, and that the effect was mediated by trypophobia. Experiment 2 replicated Experiment 1 using images of human face. The results showed that, as in Experiment 1, a significant mediation effect of trypophobia was obtained, although the relationship between social anxiety and the discomfort rating was stronger than in Experiment 1. Our findings suggest that both social anxiety and trypophobia contribute to the induction of discomfort when one is gazed at by many people. 


\section{Fear of eyes: Triadic relation among social anxiety, trypophobia, and}

6 *Corresponding author: Kengo Chaya

7 Address: Graduate School of Human-Environment Studies, Kyushu University,

8 6-19-1 Hakozaki, Higashi-ku, Fukuoka 812-8581 Japan

9 E-mail: kengo.chaya.882@s.kyushu-u.ac.jp

10 TEL \& FAX: +81-92-642-2418 


\section{Abstract}

12 Imagine you are being gazed at by multiple individuals simultaneously. Is the provoked anxiety a

13 learned social-specific response or related to a pathological disorder known as trypophobia? A

14 previous study revealed that spectral properties of images induced aversive reactions in observers

15 with trypophobia. However, it is not clear whether individual differences such as social anxiety

16 traits are related to the discomfort associated with trypophobic images. To investigate this issue,

17 we conducted two experiments with social anxiety and trypophobia and images of eyes and faces.

18 In Experiment 1, participants completed a social anxiety scale and trypophobia questionnaire

19 before evaluation of the discomfort experienced upon exposure to pictures of eye. The results 20 showed that social anxiety had a significant indirect effect on the discomfort associated with the 21 eye clusters, and that the effect was mediated by trypophobia. Experiment 2 replicated 22 Experiment 1 using images of human face. The results showed that, as in Experiment 1, a 23 significant mediation effect of trypophobia was obtained, although the relationship between 24 social anxiety and the discomfort rating was stronger than in Experiment 1. Our findings suggest 25 that both social anxiety and trypophobia contribute to the induction of discomfort when one is 26 gazed at by many people.

27 Keywords: Trypophobia, Social anxiety, Gaze perception 
29 Trypophobia is an irrational fear of holes. Typically, trypophobic images are composed of holes

30 such as the seed head of the lotus flower or a honeycomb. According to the trypophobia website

31 (www.trypophobia.com), the word "trypophobic" is relatively new. Therefore, a well-accepted

32 definition has yet to be given.

Trypophobic images, as currently understood, usually present hole-like patterns.

34 However, in one pioneering study of trypophobia conducted by Cole and Wilkins (2013), it was

35 found that the images that give rise to aversive reactions are not always holes. They explained

36 this phenomenon in terms of the "visual system as a spatial frequency analyzer" (Maffei \&

37 Fiorentini, 1973) based on the fact that trypophobic images contain relatively high contrast at

38 midrange spatial frequencies. Although this spectral feature is not a sufficient condition for

39 eliciting trypophobic aversion, as indicated by Cole and Wilkins, they found that even normal

40 individuals are more sensitive and averse to trypophobic images than normal images. That is,

41 trypophobia is not limited to being a pathological phenomenon but may be a normal visual

42 preferential tendency. As is well known, spatial frequencies influence perception in many

43 domains such as visual illusions (Giora \& Gori, 2010) or esthetic pleasure (Vannucci et al. 2014).

44 For example, the Ouchi illusion (Ouchi, 1977) is clearly related to the spatial frequency of stimuli

45 (Ashida, 2002) and fixational eye movements (Rucci et al. 2007), which are necessary for

46 avoiding perceptual fading (e.g., Martinez-Conde et al. 2006; 2013; Costela et al. 2013; McCamy

47 et al. 2014).

48 Furthermore, Cole and Wilkins (2013) showed that the spectral properties shared by

49 images of highly poisonous animals (such as the box jellyfish and the King cobra snake) are

50 similar to those of trypophobic images. The finding supported the hypothesis that humans have

51 developed the ability to detect stimuli based on an early, fast-reacting visual mechanism and that 
52 human survival has heavily relied on this mechanism to avoid potential threats from the

53 surrounding environment throughout the history of human evolution. Le, Cole, and Wilkins

54 (2015) developed the Trypophobia Questionnaire (TQ). Thanks to the TQ, researchers have been

55 able to investigate the individual differences in the trypophobic trait. Le, Cole, and Wilkins also

56 showed that convex objects induce aversion as effectively as concave objects. This result

57 supports the above investigation in that the spectral properties possessed by images are important

58 in inducing trypophobic reactions. However, the above studies focused on only the perceptual

59 aspect of trypophobia, but the underlying cognitive mechanism has not been revealed: Does the

60 aversion to trypophobic images depend on the other personal traits of observers?

61 In the present study, we focused on social anxiety. Social anxiety disorder is the fear

62 of a social situation that may involve negative judgment from others. Social anxiety disorder is a

63 relatively common psychiatric disorder with a lifetime prevalence of $10 \%$ to $15 \%$ (Kessler et al.,

64 2005). Previous studies revealed that people with social anxiety disorder have a fear of eye

65 contact or being gazed at. For instance, the gaze cone for patients with social anxiety disorder is

66 enlarged in the presence of other observers (Gamer et al., 2011). The more socially anxious a

67 person is, the more frequently he or she feels that he or she is being looked at by others and report

68 reactive behavior, such as fear and avoidance of eye contact (Schneier et al., 2011; Schulze et al.,

69 2013). A study conducted in a visual-reality environment showed that individuals with social

70 anxiety disorder are more likely to be distressed when they have to perform a speech in front of

71 an audience (Cornwell et al., 2011). Individuals with social anxiety disorder also showed a longer

72 visual scan path and greater total fixation time at the non-social regions of the display in between

73 and around the audience's faces during a speech (Chen et al., 2015). Another study (Moukheiber

74 et al., 2010) showed that avoidant patterns of eye, such as the number of fixations and total

75 fixation duration, decreased significantly for social phobic patients when they were presented 
76 with negative expressions (such as anger or disgust).

77 Considering the above studies, we hypothesized that social anxiety has an influence on

78 the degree of discomfort with trypophobic images composed of clusters of human eyes and faces.

79 The aim of the current study is to investigate three questions. The first question is whether images

80 that contain clusters of human eyes and faces can induce aversion. The second question is

81 whether the TQ shows validity and reliability in other populations, as suggested by the previous

82 study (Le et al., 2015). Third, we are interested in whether social anxiety directly involves

83 discomfort due to the trypophobic images composed of clusters of human eyes and faces. For

84 these purposes, we cropped the eye and face images separately. The relationships among the TQ, 85 the Leibowitz Social Anxiety Scale (LSAS; Liebowitz, 1987), and the Discomfort Rating Score

86 (DRS) were analyzed accordingly. In Experiment 1, we examined these questions with English 87 and Japanese speakers. In Experiment 2, we tested whether a cluster of faces also induced 88 discomfort in the same manner as cropped eyes.

\section{Experiment 1}

90 Method

91 Participants

92 The experiment was conducted online using Qualtrics (Provo, 2015). Participants whose

93 native language was either English or Japanese were recruited from Qualtrics panels and received

94 the designated compensation for each attempt. For those who made multiple attempts, only the 95 first attempt was recorded. Eighty-seven English speakers took part in the study via the English 96 section (66 males and 21 females; mean age $=18.8$ yrs., $\mathrm{SD}=11.1$ yrs.). The Japanese-speaking 97 group comprised one hundred and twenty-two individuals (71 males and 51 females; mean age = 9825.3 yrs., $\mathrm{SD}=9.6$ yrs.). The purpose of the present study was not revealed to the participants. The 
99 experiments were conducted according to the principles of the Helsinki Declaration. The ethical

100 committees of Kyushu University approved the protocol (approval number: 2013-008). Prior to

101 the experiments, the participants consented to participation in the survey, and they could quit at

102 any time if they felt sick due to the observation of disgusting images.

\section{Apparatus, Stimuli, and Procedure}

104 The survey consists of three parts: the social anxiety scale, the Trypophobia scale, and 105 the discomfort rating of eyes. The order of these three scales was randomized across all 106 participants. There was no time limit to complete each scale.

107 Liebowitz social anxiety scale: We used the English version of the LSAS (Liebowitz, 1987) or

108 the Japanese version of the scale (Asakura et al., 2002) according to the participants' language 109 background. This scale consists of two sections. In the "fear or anxiety" section, the questions 110 asked "how anxious or fearful do you feel in the specific situation." In this section, each item was 111 scored on a 4-point Likert-scale: "None," "Mild," "Moderate" and "Severe." The second section 112 was the "avoidance" section, in which the questions asked "how often do you avoid the 113 situation." In this section, each item was scored on a 4-point Likert-scale: "Never (0\%)," 114 “Occasionally (1-33\%)," "Often (33-67\%)" and "Usually (67-100\%)." The order of items was 115 randomized across sections.

116 Trypophobia scale: We employed the original English version of the TQ, and the images of a 117 lotus seed head and a honeycomb for all participants from the previous study (Le et al., 2015).

118 Moreover, we developed a Japanese version of the TQ by directly translating items from the 119 original version into Japanese, and we confirmed its literal comprehensiveness by back120 translation with the original authors. The English and Japanese versions each contained 19 items; 121 each item was rated based on a 10-point Likert-scale from 1 (strongly disagree) to 10 (strongly 
122 agree).

123 Rating of Eye Cluster: We exported the real eyes stimuli from the database of ATR-Promotions

124 (DB99; ATR-Promotions, 2006). We extracted only the eyes with their fringes and unified the

125 size for each eye picture using GIMP2.8.14 (www.gimp.org). In the real eye block, there were

126 two variables: Pair (single and paired) and Number of eyes (1, 4, 16, and 64; Figure 1).

127 Participants viewed each eye stimulus and evaluated it using a DRS from 0 (not at all 128 uncomfortable) to 10 (extremely uncomfortable). The order of the stimulus presentation was

129 randomized for each participant. Each eye stimulus was presented two times, so participants 130 viewed 16 trials.

\section{Results}

\section{The effect of eye types on the perceived discomfort}

134 We performed analyses of variance (ANOVAs) to determine whether the number and 135 pair of eyes affected the participants' DRS. A three-way within-participant ANOVA with 136 language (English and Japanese), pair (single and paired), and number of eyes (4, 16, and 64) as

137 factors was conducted. To discriminate clearly whether the number of eyes or the pair condition 138 affected the DRS, the one- and two-eye conditions were excluded from the analysis to match the 139 total number of eyes between the single and paired conditions.

$\left.141206)=41.16, p<.001, \eta_{\mathrm{p}}{ }^{2}=.17\right)$, pair $\left(F(1,206)=25.57, p<.001, \eta_{\mathrm{p}}{ }^{2}=.11\right)$ and number of eyes

$142\left(F(2,412)=151.86, p<.001, \eta_{\mathrm{p}}^{2}=.42\right)$. Multiple comparisons using Ryan's method revealed

143 that the 64-eye condition was significantly perceived to be more uncomfortable than the 4- and 144 16-eye conditions, and the 16-eye condition was significantly perceived to be more 
145 uncomfortable than the 4-eye condition. An interaction between language and number of eyes

146 was significant $\left(F(2,412)=4.32, p=.014, \eta_{\mathrm{p}}{ }^{2}=.020\right)$. Tests of simple main effect revealed that

147 the DRSs of Japanese speakers were significantly higher than the DRSs of English speakers in all

148 eye conditions (4-eyes: $F(1,618)=29.58, p<.001 ; 16$-eyes: $F(1,618)=39.24, p<.001 ; 64$ -

149 eyes: $F(1,618)=46.46, p<.001)$. For both language groups, the 64-eye condition was 150 perceived to be significantly more uncomfortable than the 4- and 16-eye conditions, and the 16-

151 eye condition was perceived to be significantly more uncomfortable than the 4-eye condition 152 (English: $F(2,412)=52.59, p<.001$; Japanese: $F(2,412)=103.60, p<.001)$. The other 153 interactions were found to be not significant (language and pair: $F(1,206)=2.63, p=.11$; pair 154 and number of eyes: $F(2,412)=1.65, p=.19$; language, pair and number of eyes: $F(2,412)=$ $1551.23, p=.29)$.

\section{Validity and reliability of $T Q$}

158 To demonstrate the validity and reliability of the TQ, a confirmatory factor analysis 159 (with promax rotation, Maximum-likelihood method) was individually conducted on the English 160 and Japanese TQ scores. As in a previous study (Le et al., 2015), a scree plot showed that a one

161 factor solution was sufficient to explain all variances in the analysis for both language versions of 162 the TQ. The results are shown in Tables 1 and 2. The factor loading value of each item, $\alpha$ 163 coefficients, factor contribution and cumulative contribution are approximately consistent with 164 those of Le et al. (2015). In the following analysis, we used a sum of the item scores on the TQ 165 and the LSAS (Liebowitz, 1987).

--Table 1 around here-- 
168

169

170

171 The correlations among the DRS (single and paired), the TQ score, and the LSAS score are

172 shown in Table 3. There were significant correlations between the TQ score and the DRS in the

173 single and paired conditions. Moreover, the correlations between the LSAS score and the DRS in 174 both the single and paired conditions were also significant.

175

176

177

178

179

180

181

182

183

184

185

186

187

188

189

\section{Correlation among DRS, TQ score and LSAS score}

We calculated the DRS by summing the scores from each pair condition (single and paired) across all participants (i.e., data from both language groups were analyzed altogether). --Table 3 around here--

\section{Mediation effect of $T Q$}

To investigate the direct influence of the LSAS score on the DRS, we conducted a mediation analysis. We set the LSAS and TQ scores as predictors of the two conditions (single \& paired) used in the correlation analysis. The mediation model is shown in Figure 4, and the results are shown in Table 4 . In both the single and paired conditions, the path from the LSAS score to the DRS was significant (single: $\beta=.15, p=.034$; paired: $\beta=.19, p=.006$ ). When the TQ score was set as a mediator, the path from the LSAS score to the TQ score was significant (single: $\beta=.27, p<.001$; paired: $\beta=.27, p<.001$ ) and the path from the TQ score to the DRS was also significant (single: $\beta=.38, p<.001$; paired: $\beta=.41, p<.001$ ). However, the path from the LSAS score to the DRS was not significant (single: $\beta=.044, p=.51$; paired: $\beta=.078, p=$. 23). A Sobel test revealed that the mediation effects were significant (single: $Z=3.31, p=.001$; paired: $Z=3.45, p=.001)$. 
190 Discussion

191 In Experiment 1, we examined the relationship between social anxiety and the 192 discomforting symptoms induced by images of eye clusters. In line with a previous study (Le et 193 al., 2015), we found that not only clusters of holes but also the images that contain clusters of 194 other objects induce aversive reactions. Cultural differences might be a factor in the discrepancy 195 of the aversive cognition because we found that Japanese speakers experienced more discomfort 196 than English speakers.

197 The results from the factor analysis also supported the previous study (Le et al., 2015).

198 The analysis showed that all items consisted of one common factor and satisfied the criterion for 199 acceptable loadings for both the English and Japanese scales. In addition, the analysis indicates 200 that direct translation of the TQ does not affect its validity or reliability when the TQ is applied to 201 a study on another population.

202 We calculated the correlations among the DRS, the LSAS score, and the TQ score, from 203 which we found a positive correlation $(\mathrm{r}=.27)$ between the LSAS and TQ scores. Le et al. (2015) 204 showed a small correlation $(\mathrm{r}=.21)$ between the TQ and the State-Trait Anxiety Inventory (STAI; 205 Spielberger, 1983) and suggested that general anxiety accounts for trypophobia. Our results in 206 Experiment 1 supported their findings.

207 The mediation analysis showed a significant mediation effect when the TQ score was a 208 mediator; however, the path from the LSAS score to the DRS was not significant. These results 209 were contrary to our hypothesis that there is positive correlation between social anxiety and the 210 DRS concerning trypophobic images composed of eye clusters.

211 One of the reasons the significant path between social anxiety and discomfort was not 212 observed may be attributed to the stimulus property. In the previous studies that investigated the 213 relationship between social anxiety and gaze perception, stimulus eyes were usually embedded in 
214 a face (Gamer et al., 2011; Schulze et al., 2013). Moreover, faces are processed holistically in

215 specialized brain areas such as the fusiform face area (Andrews, Davies-Thompson, Kingstone, \&

216 Young, 2010; Arcurio, Gold, \& James, 2012; Schiltz \& Rossion, 2006). Thus, it is possible that if

217 the individuals with a high social anxiety trait show a strong avoidance reaction to the gaze

218 specifically when the eyes appear in a face, the direct effect from the LSAS score to the DRS

219 may be weak because, in Experiment 1, the eyes were not in a face. Hence, in Experiment 2, we

220 used the trypophobic images containing faces with eyes and investigated whether social anxiety

221 could directly involve the aversion to images of gazing faces. If so, we could obtain a significant

222 direct effect from the LSAS score to the DRS even after mediation by the TQ score.

\section{Experiment 2}

\section{Method}

\section{Participants}

226 In this experiment, a new sample of 499 English speakers was recruited from Qualtrics

227 panels (159 females and 340 males; mean age $=34.6$ yrs., $\mathrm{SD}=9.8 \mathrm{yrs}$.). The purpose of the 228 present study was not revealed to the participants.

\section{Stimuli and procedure}

230 The procedure was identical to Experiment 1, except that the rating of eye clusters was 231 replaced by the rating of face clusters. As facial stimuli, we selected 32 neutral faces (half males, 232 half females; AF01NES AF16NES, AM01NES AM11NES, AM13NES AM15NES, 233 AM17NES, AM18NES) from the Karolinska Directed Emotion Face Set (Lundqvist, Flykt, \& 234 Öhman, 1998). To exclude hair, we cropped these face stimuli to elliptical shapes and unified the 235 size of each face picture. 
237 4, 16 and 64; Figure 4). The order of the stimuli presentation was randomized for each 238 participant. Only English versions of the scales were used in this experiment because all the 239 participants were English speakers.

--Figure 4 around here--

241 Results

242 The effect of face conditions on perceived discomfort

243 As shown in Figure 5, for the data in the cluster of faces, a one-way within-participant

244 ANOVA with the numbers of faces (2, 8, and 32) as factors was conducted, which revealed 245 significant main effects associated with the number of faces $\left(F(2,996)=165.87, p<.001, \eta_{\mathrm{p}}^{2}=\right.$. 246 24). The multiple comparisons revealed that the 32-face condition was significantly more

247 uncomfortable than the 4- and 16-face conditions and that the 16-face condition was significantly 248 more uncomfortable than the 4-face condition (all $p s<.001$ ).

--Figure 5 around here--

250

251

252

\section{Validity and reliability of $T Q$}

A confirmatory factor analysis (with promax rotation, Maximum-likelihood method) was individually conducted on the TQ scores. As shown in Table 5, all the items constituted one common factor. The factor loading values of each item, $\alpha$ coefficients, factor contribution and cumulative contribution were similar to those of both Le et al. and Experiment 1. In the following analysis, we used a sum of the item scores as the trypophobia scale score.

$$
\text { --Table } 5 \text { around here-- }
$$

\section{Correlation among DRS, TQ and LSAS scale}

$$
\text { We calculated the DRS by summing the scores of each of the faces for each participant. Table }
$$


2596 shows the correlations of the DRSs and the TQ and LSAS scores. There were significant 260 correlations among the DRSs and the TQ and LSAS scores.

261

--Table 6 around here--

262 Mediation effect of $T Q$

263 In the mediation analysis, we entered the LSAS and TQ scores as predictors of the DRS of 264 faces. The mediation model was set as in Experiment 1, and the results are shown in Table 7. The 265 path from the LSAS score to the DRS was significant $(\beta=.36, p<.001)$. When the TQ score was 266 a mediator, the path from the LSAS score to the TQ score was significant $(\beta=.32, p<.001)$ and 267 the path from the TQ score to the DRS was also significant $(\beta=.42, p<.001)$. The path from the 268 LSAS score to the DRS was still significant, although the $\beta$ score decreased $(\beta=.23, p<.001)$. A 269 Sobel test revealed the significant mediation effect $(Z=6.14, p<.001)$.

270

--Table 7 around here--

\section{Discussion}

272 We examined whether social anxiety directly linked to aversion to trypophobic images 273 composed of faces in Experiment 2. The results revealed that the cluster of faces induced 274 aversion and that the DRS increased when the number of faces increased; the validity and 275 reliability of TQ was confirmed, as in Experiment 1. These results were in agreement with 276 Experiment 1 and supported previous findings (Le et al., 2015).

277 In addition, the correlation analysis showed that the correlation coefficient between the 278 LSAS score and the DRS was not higher than that between the TQ score and the DRS. The 279 results suggested that even when the eyes were embedded in a face, social anxiety was not 280 strongly related to discomfort for facial clusters, although the correlation was significant. 
282 score to the DRS was significant in Experiment 2 when the TQ score was set as a mediator. This

283 significant direct effect after mediation was not shown in Experiment 1, where we used only the 284 cropped eye images. Based on these results, it is suggested that the perception of a face 285 strengthens the induction of discomfort to gaze in individuals with social anxiety disorder and 286 that trypophobia mediates this relation.

The present study aimed to investigate whether social anxiety is related to discomfort induced by clusters of eyes (Experiment 1) and faces (Experiment 2). The results suggested that 290 both eyes and faces induced discomfort as the number of images increased. Additionally, this 291 effect was strongly related to the social anxiety trait and was mediated by the trypophobia trait. 292 Moreover, the social anxiety trait more strongly predicted discomfort for the clusters of eyes 293 when the eyes appeared in a face.

Although our hypothesis that social anxiety is directly linked to discomfort due to the 295 trypophobic images of human face clusters was supported, we did not expect the significant 296 mediation effect in both experiments. It does not necessarily mean that only the TQ score 297 mediated the path between the LSAS score and the DRS because general anxiety may have an 298 influence on trypophobia. Nevertheless, we cannot completely exclude the possibility that the 299 relationship between the LSAS score and DRS was mediated by the TQ score. For example, 300 Moukheiber et al. (2012) showed that gaze avoidance and fear of blushing occurred with 301 individuals who have a social anxiety disorder, which seems to support the hypothesis that social 302 anxiety is a heterogeneous disorder. Further investigations are needed to clarify whether 303 trypophobia can be classified as a subtype of social anxiety disorder based on our findings that 304 the TQ score mediated the LSAS score and the DRS. 
306 and cultures were not examined. For example, in Experiment 1, the results of the ANOVA 307 showed that Japanese speakers feel more discomfort than English speakers. However, the 308 question of whether the difference in the DRS between Japanese and English speakers is caused 309 by the aversion to trypophobic images or the sensitivity to gaze has not yet been determined. If 310 the cultural difference in DRS was specific to the trypophobic images of eye clusters, one can 311 argue that the difference came from cultural or ethnic differences in the sensitivity to gaze. This 312 hypothesis predicts that Japanese speakers would show stronger social anxiety than English 313 speakers, because individuals with social anxiety tend to attend to gaze (Schneier et al., 2011;

314 Schulze et al., 2013). But this was not the case. Experiment 1 showed that the LSAS score of the 315 English speakers was significantly higher than that of the Japanese speakers $(\mathrm{M}=61.63$ vs. 316 54.46). Moreover, considering counter-evidence that social anxiety symptoms are more likely to 317 be found in Japanese than American (e.g., Dinnel, Kleinknecht, \& Tanaka-Matsumi, 2002), it is 318 bold to conclude that the DRS simply reflected the difference in the LSAS scores. Another 319 possible explanation is that the spatial frequency information to evaluate discomfort depends on 320 cultures. For example, previous studies revealed that social anxiety, culture, and emotion 321 modulated the mental and neural processing of spatial frequency information or facial recognition 322 (Curby, Johnson, \& Tyson, 2012; Miellet, Vizioli, He, Zhou, \& Caldara, 2015; Riwkes, 323 Goldstein, \& Gilboa-Schechtman, 2015; Wieser \& Moscovitch, 2015). Based on the fact that 324 the esthetic pleasure was affected by spatial frequencies in the Italian and Japanese observers 325 (Vannucci et al. 2014), it is premature to conclude that such influence of spatial frequency is 326 specific to face. Possibly, the range of spatial frequency information to induce discomfort may be 327 a common between individuals with social anxiety disorder and trypophobia. Therefore, the 328 relationship between trypophobia and cultural differences or individual differences in anxiety 
329 should be further investigated on the perspective of spatial characteristic of visual stimuli in the 330 future.

331 In conclusion, the current study revealed that not only clusters of holes but also clusters 332 of other objects such as eyes and faces induce aversive reactions. The validity and reliability of 333 the TQ and the fact that social anxiety directly involved the aversion to trypophobic images of 334 faces were also established. Further studies are required to explore other factors that influence 335 trypophobia. Such studies could contribute to understanding why some individuals develop an 336 aversion to trypophobic images but others do not. 


\section{Acknowledgements}

supports for developing the Japanese version of the Trypophobia Questionnaire. 


\section{References}

341 Andrews, T. J., Davies-Thompson, J., Kingstone, A., \& Young, A. W. (2010). Internal and external features of the face are represented holistically in face-selective regions of visual cortex. The Journal of Neuroscience, 30(9), 3544-3552. http://doi.org/10.1523/JNEUROSCI.4863-09.2010

345

346

347

348

349

350

351

352

353

354

355

356

357

358

359

360

361

362

363

364 single face parts and part combinations. Neuropsychologia, 50(10), 2454-2459. http://doi.org/10.1016/j.neuropsychologia.2012.06.016

Asakura, S., Inoue, S., Sasaki, F., Sasaki, Y., Kitagawa, N., Inoue, T., Denda, K., Ito, M., Matsubara, M., \& Koyama, T. (2002). Reliability and validity of the Japanese version of the Liebowitz social anxiety scale. Clinical Psychiatry, 44(10), 1077-1084.

Ashida, H. (2002). Spatial frequency tuning of the Ouchi illusion and its dependence on stimulus size. Vision Research, 42(11), 1413-1420. doi:10.1016/S0042-6989(02)00064-0

ATR-Promotions (2006). ATR facial expression database DB99 [Data file]. http://www.atrp.com/products/face-db.html

Chen, N. T., Thomas, L. M., Clarke, P. J., Hickie, I. B., \& Guastella, A. J. (2015). Hyperscanning and avoidance in social anxiety disorder: the visual scanpath during public speaking. Psychiatry Research, 255, 667-672. doi:10.1016/j.psychres.2014.11.025

Cole, G. G., \& Wilkins, A. J. (2013). Fear of holes. Psychological Science, 24(10), 1980-1985. doi:10.1177/0956797613484937

Cornwell, B. R., Heller, R., Biggs, A., Pine, D. S., \& Grillon, C. (2011). Becoming the center of attention in social anxiety disorder; Startle reactivity to a virtual audience during speech anticipation. The Journal of Clinical Psychiatry, 72(7), 942-948. doi:10.4088/JCP.09m05731blu 
Microsaccades restore the visibility of minute foveal targets. PeerJ. doi:10.7717/peerj.119

367 Curby, K. M., Johnson, K. J., \& Tyson, A. (2012). Face to face with emotion: Holistic face processing is modulated by emotional state. Cognition and Emotion, 26(1), 93102. doi:10.1080/02699931.2011.555752

370 Gamer, M., Hecht, H., Seipp, N., \& Hiller, W. (2011). Who is looking at me? The cone of gaze widens in social anxiety. Cognition and Emotion, 25(4), 756-764. doi:10.1080/02699931.2010.503117

373 Giora, E., \& Gori, S. (2010). The perceptual expansion of a filled area depends on textural characteristics. Vision Research, 50(23), 2466-2475. doi:10.1016/j.visres.2010.08.033

375 Dinnel, D. L., Kleinknecht, R. A., \& Tanaka-Matsumi, J. (2002). A Cross-Cultural Comparison of 376 75-84. doi:10.1023/A:1015316223631

Kessler, R. C., Berglund, P., Demler, O., Jin, R., Merikangas, K. R., \& Walters, E. E. (2005). Lifetime prevalence and age-of-onset distributions of DSM-IV disorders in the National Comorbidity Survey Replication. Archive General Psychiatry. 62(6), 593602. doi:10.1001/archpsyc.62.6.593

Le, A. T. D., Cole, G. G., \& Wilkins, A. J. (2015). Assessment of trypophobia and an analysis of 384 its visual perception. The Quarterly Journal of Experimental Psychology, doi:10.1080/17470218.2015.1013970.

Liebowitz, M. R. (1987). Social anxiety. Modern Problems of Pharmacopsychiatry, 22, 141-173.

Lundqvist, D., Flykt, A., \& Öhman, A. (1998). The Karolinska Directed Emotional Faces 388 KDEF, CD ROM from Department of Clinical Neuroscience, Psychology section,

389 Maffei, L., \& Fiorentini, A. (1973). The visual cortex as a spatial frequency analyser. Vison 
391 Martinez-Conde, S., Macknik, S. L., Troncoso, X. G., \& Dyar, T. A. (2006). Micorosaccades counteract visual fading during fixation. Neuron, 49(2), 297-305. doi:10.1016/j.neuron.2005.11.033

394 Martinez-Conde, S., Otero-Millan, J., \& Macknik, S. L. (2013). The impact of microsaccades on

395

396

397 398

399

400 401 402 403

404 405 406 407 408 409 410 411 412 Schulze, L., Renneberg, B., \& Lobmaier, J. S. (2013). Gaze perception in social anxiety and 413 vision: Towards a unified theory of saccadic function. Nature Reviews Neuroscience, 14, 83-96. doi:10.1038/nrn3405

McCamy, M. B., Macknik, S. L., Martinez-Conde, S. (2014). Different fixational eye movements mediate the prevention and the reversal of visual fading. The Journal of Physiology, 592(19), 4381-4394. doi: 10.1113/jphysiol.2014.279059

Miellet S, Vizioli L, He L, Zhou X and Caldara R (2013) Mapping face recognition information use across cultures. Front. Psychol. 4:34. doi: 10.3389/fpsyg.2013.00034

Moukheiber, A., Rautureau, G., Perez-Diaz, F., Soussignan, R., Dubal, S., Jouvent, R., \& Pelissolo, A. (2010). Gaze avoidance in social phobia: objective measure and correlates. Behaviour Research and Therapy, 48(2), 147-151. doi:10.1016/j.brat.2009.09.012

Schiltz, C., \& Rossion, B. (2006). Faces are represented holistically in the human occipitotemporal cortex. $\quad$ NeuroImage, 32(3), 1385-1394. http://doi.org/10.1016/j.neuroimage.2006.05.037

Schneier, F. R., Rodebaugh, T. L., Blanco, C., Lewin, H., \& Liebowitz, M. R. (2011). Fear and avoidance of eye contact in social anxiety disorder. Comprehensive Psychiatry, 52(1), 81-87. doi:10.1016/j.comppsych.2010.04.006 social anxiety disorder. Frontiers in Human Neuroscience, 7. 
414 doi:10.3389/fnhum.2013.00872

415 Spielberger, C. D. (1983). State-trait anxiety inventory for adults. Palp Alto, CA: Mind Garden.

416 Riwkes, S., Goldstein, A., \& Gilboa-Schechtman, E. (2015). The temporal unfolding of 417 face processing in social anxiety disorder - a MEG study. Neurolmage: Clinical, $418 \quad$ 7, 678-687. doi:10.1016/j.nicl.2014.11.002

419 Rucci, M., Iovin, R., Poletti, M., \& Santini, F. (2007). Miniature eye movements enhance fine 420 spatial detail. Nature, $447,852-855$. doi:10.1038/nature05866

421 Vannucci, M., Gori, S., \& Kojima, H. (2014). The spatial frequencies influence the aesthetic 422 judgment of buildings transculturally. Cognitive Neuroscience, 5(3-4), doi: $423 \quad 10.1080 / 17588928.2014 .976188$

424 Wieser, M. J., \& Moscovitch, D. A. (2015). The Effect of Affective Context on Visuocortical 425 Processing of Neutral Faces in Social Anxiety. Front. Psychol. 6:1824. doi: $10.3389 /$ fpsyg.2015.01824 
427

428

429 430 431 432 433 434 435 436 Experiments 1 and 2.

437 438 439 the real eye image.

\section{Figure legends}

Figure 1. The examples of stimuli used in Experiment 1. The letter of each label indicates the Pair-condition (single or paired), and the number in each label indicates the Number-of-eyes-condition $(1,4,16$, or 64$)$. The $256 \times 256$ pixels of the real eye image were created using the $64 \times 64$ pixels of one eye in the 1 - and 16-eye-condition. The 4 -eye-condition used the $128 \times 128$ pixels of the real eye image. The 64 -eye condition used the $512 \times 512$ pixels of

Figure 2. The DRS for real eye in each condition in Experiment 1.

Figure 3. The mediation model among the LSAS score, the TQ score, and the DRS in

Figure 4. Examples of the stimuli used in Experiment 2. Each face image was scaled to an elliptical shape, $57 \times 75$ pixels.

Figure 5. The DRS for faces in each condition in Experiment 2. 
a. (1)

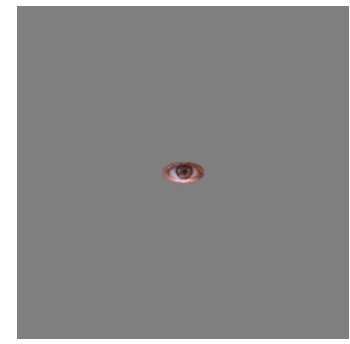

b. (1)

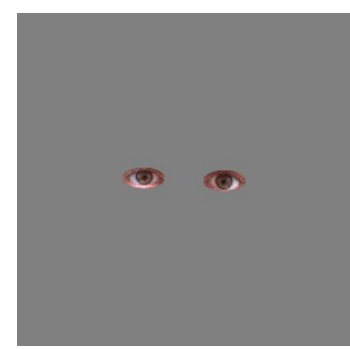

(2)

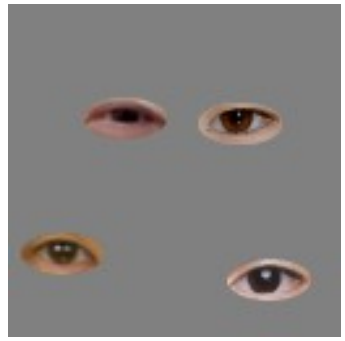

(2)

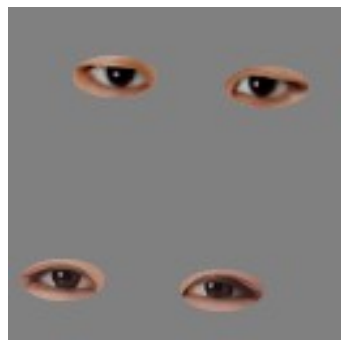

(3)

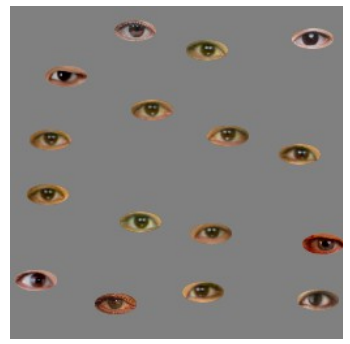

(3)

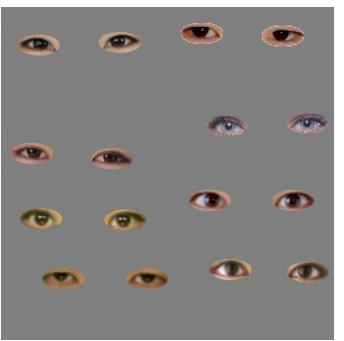

(4)

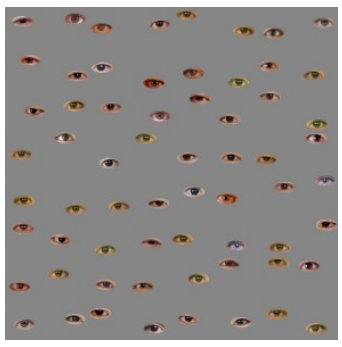

(4)

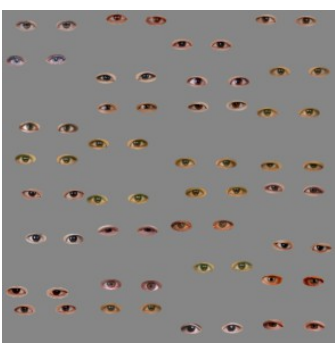

Figure 1 


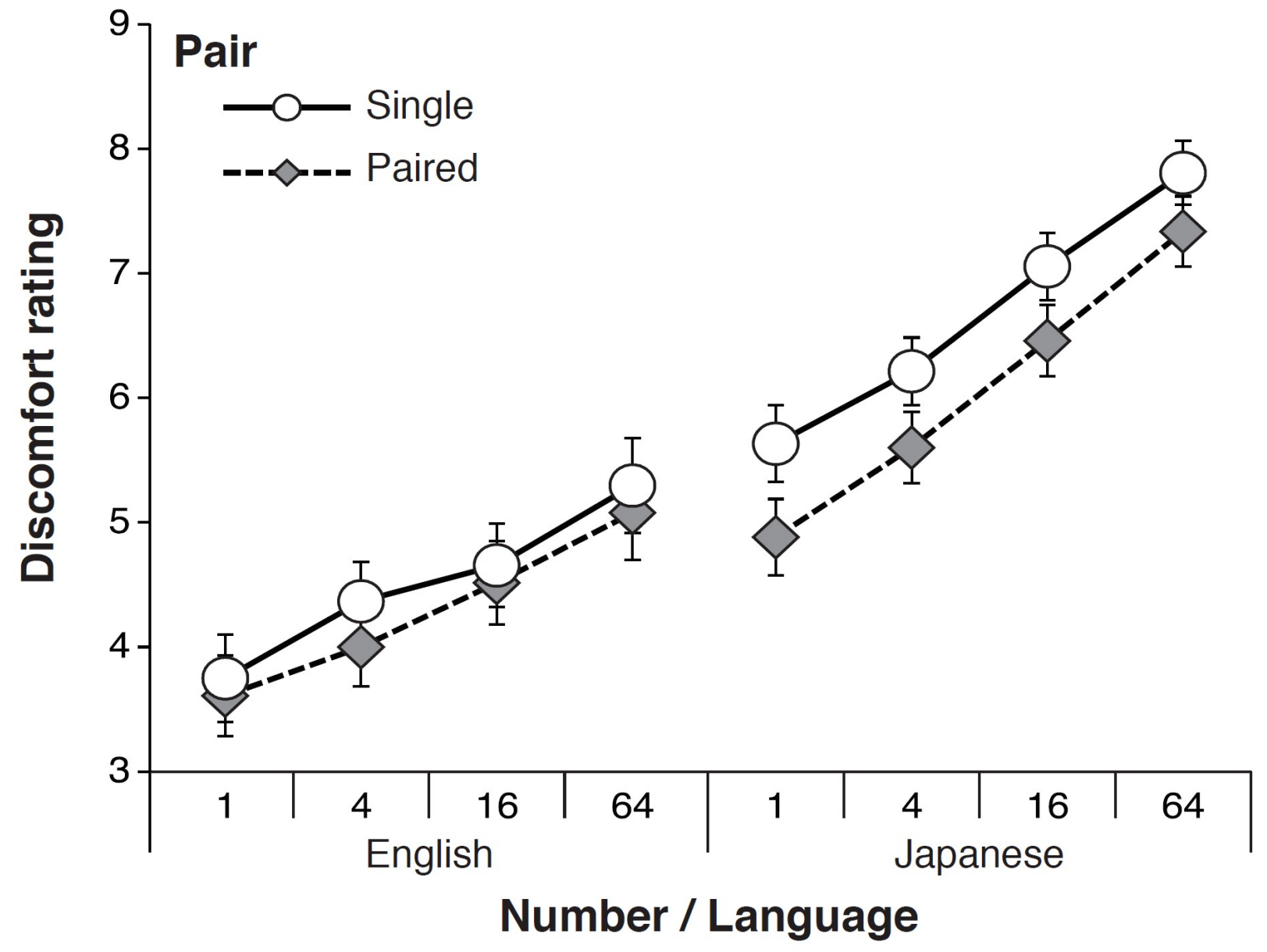

Figure 2 


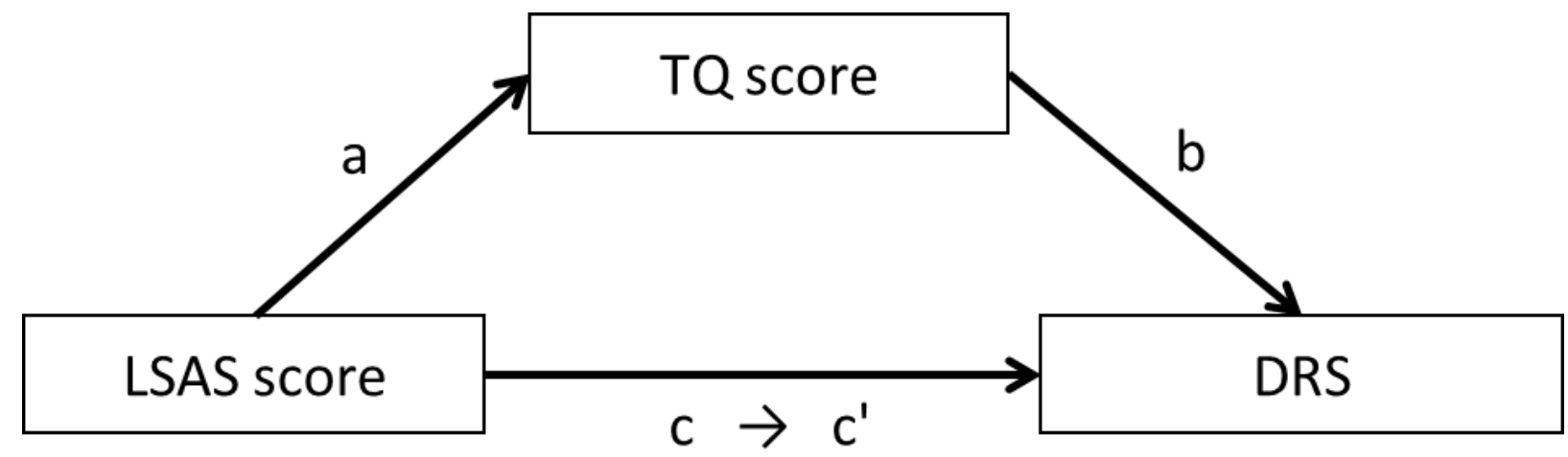

Figure 3 
(1)

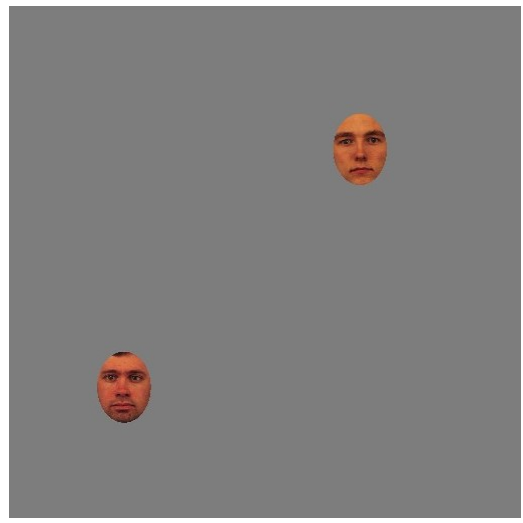

(2)

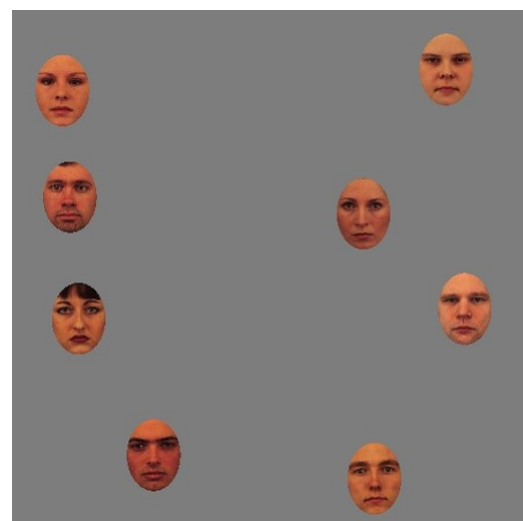

(3)

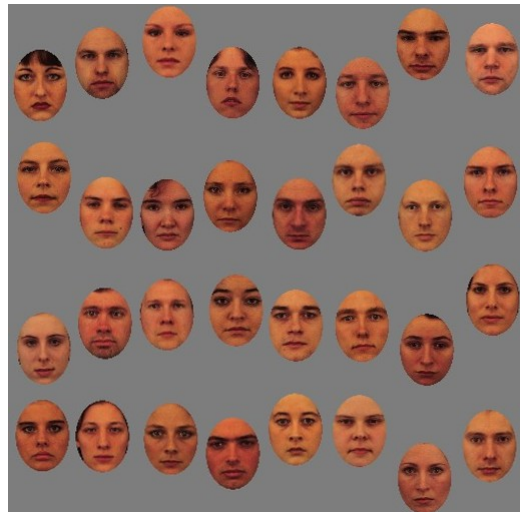

Figure 4 


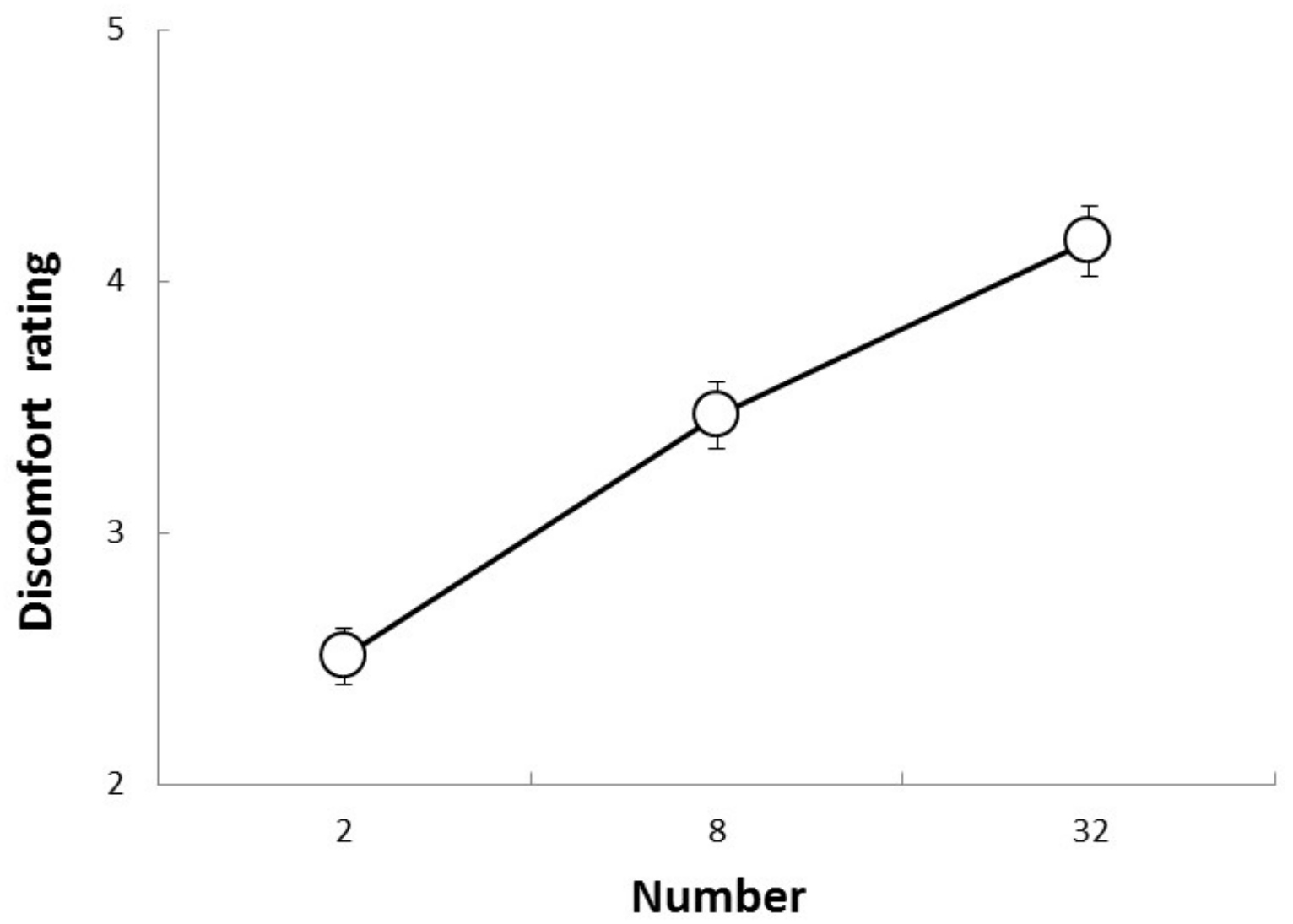

Figure 5 
Table 1. Factor loadings for the items in the TQ on English after promax rotation in Experiment 1.

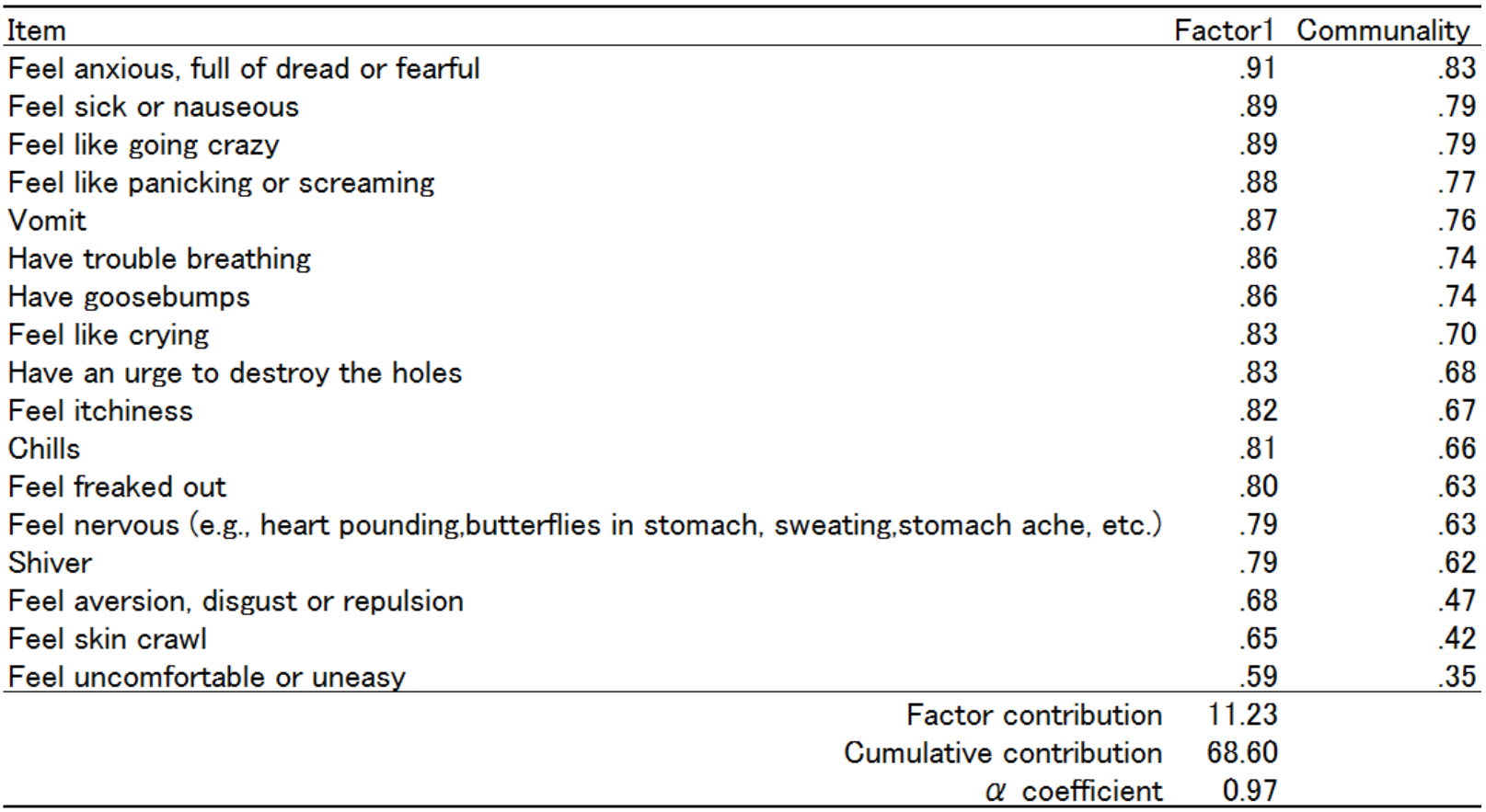


Table 2. Factor loadings for the items in the TQ on Japanese after promax rotation in Experiment 1.

\begin{tabular}{lrr}
\hline Item & Factor1 Communality \\
\hline Feel anxious, full of dread or fearful & .91 & .82 \\
Feel sick or nauseous & .89 & .80 \\
Feel nervous (e.g., heart pounding,butterflies in stomach, sweating,stomach ache, etc.) & .89 & .79 \\
Feel like panicking or screaming & .88 & .77 \\
Chills & .87 & .75 \\
Have trouble breathing & .87 \\
Have goosebumps & .86 \\
Feel like going crazy & .86 \\
Feel itchiness & .75 \\
Feel freaked out & .82 \\
Vomit & .82 \\
Feel uncomfortable or uneasy & .81 \\
Shiver & .73 \\
Feel skin crawl & .78 \\
Feel like crying & .77 \\
Feel aversion, disgust or repulsion & .67 \\
Have an urge to destroy the holes & .73 \\
\hline & .72 \\
\hline
\end{tabular}


Table 3. Correlation among DRS, TQ and LSAS score in Experiment 1

\begin{tabular}{lcccc}
\hline & 1 & 2 & 3 & 4 \\
\hline 1. DRS for single eye & - & & & \\
2. DRS for paired eyes & $.90^{* *}$ & - & & \\
3. LSAS score & $.15^{*}$ & $.19^{* *}$ & - & \\
4. TQ score & $.39^{* *}$ & $.44^{* *}$ & $.27^{* *}$ & - \\
${ }^{* *} p<.01,{ }^{*} p<.05$ & & &
\end{tabular}


Table 4. The result of mediation analysis in Experiment 1

\begin{tabular}{lccccc}
\hline DRS & Path & \multicolumn{1}{l}{ B } & \multicolumn{1}{l}{$S E$} & $\mathrm{df}$ & $t$ \\
\hline Single eye & $\mathrm{a}$ & .27 & .0933 & 206 & $4.09^{* * *}$ \\
& $\mathrm{~b}$ & .38 & .0050 & 205 & $5.63^{* *}$ \\
& $\mathrm{c}$ & .15 & .0073 & 206 & $2.13^{*}$ \\
Paired eyes & $\mathrm{c}^{\prime}$ & .04 & .0071 & 205 & 0.66 \\
& $\mathrm{a}$ & .27 & .0933 & 206 & $4.09^{* *}$ \\
& $\mathrm{~b}$ & .42 & .0048 & 205 & $6.40^{* * *}$ \\
& $\mathrm{c}$ & .19 & .0070 & 206 & $2.80^{*}$ \\
${ }^{* *} p<.01,{ }^{*} p<.05$ & $\mathrm{c}^{\prime}$ & .08 & .0067 & 205 & 1.20 \\
\hline & & & & &
\end{tabular}


Table 5. Factor loadings for the items in the TQ after promax rotation in Experiment 2.

\begin{tabular}{lrr}
\hline Item & Factor 1 Communality \\
\hline Feel sick or nauseous & .92 & .85 \\
Feel like panicking or screaming & .92 & .84 \\
Have trouble breathing & .92 \\
Feel nervous (e.g., heart pounding,butterflies in stomach, sweating,stomach ache, etc.) & .91 \\
Feel like going crazy & .91 \\
Feel anxious, full of dread or fearful & .90 \\
Feel freaked out & .90 \\
Chills & .90 \\
Have goosebumps & .90 \\
Shiver & .82 \\
Feel itchiness & .87 \\
Feel like crying & .87 \\
Have an urge to destroy the holes & .81 \\
Vomit & .87 \\
Feel uncomfortable or uneasy & .86 \\
Feel aversion, disgust or repulsion & .80 \\
Feel skin crawl & .85 \\
& .76 \\
& .76 \\
\hline
\end{tabular}


Table 6. Correlation among DRS, TQ and LSAS score in Experiment 2

\begin{tabular}{lccc}
\hline & 1 & 2 & 3 \\
\hline 1. DRS for faces & - & & \\
2. LSAS score & $.36^{* *}$ & - & \\
3. TQ score & $.56^{* *}$ & $.32^{* *}$ & - \\
${ }^{* *} p<.01,{ }^{*} p<.05$ & &
\end{tabular}


Table 7. The result of mediation analysis in Experiment 2

\begin{tabular}{lccllr}
\hline DRS & Path & \multicolumn{1}{l}{ B } & SE & df & \multicolumn{1}{c}{$t$} \\
\hline Faces & $\mathrm{a}$ & .32 & .073 & 497 & $7.58^{* *}$ \\
& $\mathrm{~b}$ & .42 & .008 & 496 & $10.47^{\text {** }}$ \\
& $\mathrm{c}$ & .36 & .015 & 497 & $8.64^{\text {** }}$ \\
& $\mathrm{c}^{\prime}$ & .23 & .014 & 496 & $5.66^{\text {** }}$ \\
${ }^{* *} p<.01,{ }^{*} p<.05$ & & & & &
\end{tabular}

